\title{
Teaching or Learning from Baby: Inducing Explicit Parenting Goals Influences Caregiver Intrusiveness
}

Lucy S. King ${ }^{1}$, Kaylin E. Hill ${ }^{3}$, Elizabeth Rangel ${ }^{2}$, Ian H. Gotlib ${ }^{1}$, Kathryn L. Humphreys ${ }^{3}$

\author{
${ }^{1}$ Department of Psychology, Stanford University, Stanford, CA \\ ${ }^{2}$ San Diego State University/University of California San Diego Joint Doctoral Program in \\ Clinical Psychology, San Diego, CA \\ ${ }^{3}$ Department of Psychology and Human Development, Vanderbilt University, Nashville, TN
}

\begin{abstract}
Funding Statement: Funding was provided by the National Institutes of Health (IHG, R21 MH111978; IHG, R21 HD090493; IHG, R37 MH101495; LSK, F32 HD105385; KEH, T32 MH1892; the National Science Foundation (LSK, Graduate Research Fellowship); the Jacobs Foundation (KLH, Early Career Research Fellowship 2017-1261-05).
\end{abstract}

Acknowledgements: We thank the team of research assistants and coordinators for their assistance in data collection, coding, and management. We gratefully acknowledge the participants for their contributions.

Author Note: Data and code are available at https://github.com/lucysking/teach_learn. Caregiver coding manual is available at https://osf.io/2gp6b/?view_only=4381f7d2a4cf40b1abcf0b41f76cdf63. The study was preregistered at https://osf.io/dt7ck/?view_only=d03597cc2ab4495ba81b748756d823a9.

Corresponding author: Kathryn L. Humphreys, Ph.D., Ed.M., 230 Appleton Place, \#552, Nashville, TN 37203; Phone: 615-343-0379; k.humphreys@vanderbilt.edu 


\begin{abstract}
Caregivers' goals influence their interactions with their children. In this preregistered study, we examined whether directing parents to teach their baby versus learn from their baby influenced the extent to which they engaged in intrusive (e.g., controlling, adult-centered rather than childcentered), sensitive, warm, or cognitively stimulating caregiving behaviors. Mothers and their 6month-old infants ( $\mathrm{N}=66 ; 32$ female infants) participated in a 10-minute "free play" interaction, coded in 2-minute epochs for degree of parental intrusiveness. Prior to the final epoch, mothers were randomly assigned to receive instructions to focus on 1) teaching something to their infant; or 2) learning something from their infant. A control group of mothers received no instructions. Analyses of within-person changes in intrusive behavior from before to after receiving these instructions indicated that mothers assigned to teach their infant increased in intrusiveness whereas mothers assigned to learn from their infant and mothers in the control group did not significantly change in intrusiveness. The study provides experimental evidence that caregivers' explicit goals to teach infants result, on average, in more controlling and adult-centered caregiving behavior.
\end{abstract}

Keywords: parenting goals; caregiving; intrusiveness; parent-child interactions

Public significance statement: Given the importance of intrusive caregiving behaviors in shaping children's long-term outcomes, we sought to understand the role of one potential predictor of caregiver intrusiveness. We conducted an experimental manipulation of caregiver's goals during a parent-child interaction to examine causal effects on caregiving behaviors. This study provides experimental evidence that goals to teach one's infant results in increased intrusiveness in caregiving interactions. 


\section{Introduction}

Parenting involves a long-lasting and intense commitment to providing appropriate care in the face of competing demands and varied information about what is optimal for one's child. During parenting, caregivers' cognitions, including their culturally-influenced goals for interacting with their children, guide their behavior (Hastings \& Grusec, 1998; Mageau, Bureau, Ranger, Allen, \& Soenens, 2016; Solomon \& George, 1996; Tamis-LeMonda, Way, et al., 2008). Contemporary industrialized societies emphasize formal education as a primary means of determining the success, and even the survival, of one's child. Therefore, it is not surprising that caregivers from these societies tend to value caregiving practices believed to promote children's early learning (e.g., preparing children for school by teaching colors, numbers, etc.), and that their endorsement of these practices is associated with their actual caregiving behavior (Hembacher \& Frank, 2020). The focus on caregivers as cultivators of children's early learning achievement may be especially pronounced in families with high socioeconomic status (Lareau, 2011). Although the goal of promoting early learning may lead caregivers to provide their child with more stimulation, this goal could also have unintended negative consequences (Grolnick, 2003). Specifically, the goal of teaching infants and young children may sometimes lead to more intrusive (i.e., adult- rather than child-centered) caregiving behavior.

Caregiving behaviors include multiple dimensions, including the degree to which a caregiver's behavior is intrusive. Caregivers rated to be high in intrusiveness have interaction patterns that are characterized by overly controlling behavior that is based on the caregiver's agenda rather than on the child's interests and needs (Grolnick \& Pomerantz, 2009). During play or task completion with infants and young children, caregiving rated as intrusive may manifest as the caregiver not allowing the child a "turn" or opportunity to respond, "taking over" the focus of the play or the task, interrupting the child's exploration, and/or over-stimulating the child (Sosinsky, Marakovitz, \& Carter, 2004). Caregivers who engage in more intrusive behavior may intend to direct their child in a manner that supports learning (Younesian, Eivers, 
Shahaeian, Sullivan, \& Gilmore, 2020); however, despite these intentions, more intrusive caregiving behaviors may in fact undermine learning over time by impeding children's exploration. For example, infants and toddlers who experience more intrusive caregiving have been found to have smaller receptive vocabulary, more difficulty solving math problems, and less pre-academic knowledge of colors, letters, and numbers at preschool-age (Cabrera, Shannon, \& Tamis-LeMonda, 2007; Dotterer, Iruka, \& Pungello, 2012), relatively poorer verbal and perceptual abilities and self-regulation at kindergarten-age (Hubbs-Tait, Culp, Culp, \& Miller, 2002a; Obradovic, Sulik, \& Shaffer, 2021), and lower intelligence quotient scores at school-age (Treyvaud et al., 2016). In contrast, children who experience more child-centered caregiving in which the child's autonomy is supported (e.g., with more following-the-lead caregiving behaviors) tend to have better cognitive outcomes (Bernier, Carlson, Deschênes, \& Matte-Gagné, 2012; Distefano, Galinsky, McClelland, Zelazo, \& Carlson, 2018).

Because more intrusive caregiving may affect children's development, it is important to understand what may be contributing to this behavior. Overall, there has been less research examining the antecedents of caregiving behaviors than their consequences. Research assessing the precursors of intrusive caregiving in infancy and young childhood has typically focused on associations with caregiver psychopathology (Gueron-Sela, Camerota, et al., 2018), caregiver race, ethnicity, or cultural orientation (Ispa et al., 2004), and family socioeconomic status (Clincy \& Mills-Koonce, 2013). Maternal depressive symptoms, for example, are associated with more intrusive behaviors and intrusive caregiving mediates the association between depressive symptoms and subsequently lower child executive function skills (GueronSela, Bedford, et al., 2018). The impact of caregiver intrusiveness on child outcomes may depend on one's cultural context, identification with specific racial/ethnic communities, socioeconomic status, or societal inequities. For example, while intrusive caregiving is generally associated with negative outcomes (i.e., lower executive functioning skills, expressive communication, inhibitory control, and intellectual functioning and higher child negativity) in U.S. 
samples (Clincy \& Mills-Koonce, 2013; Gueron-Sela, Bedford, et al., 2018; Ispa et al., 2004), findings among primarily Black/African American U.S. samples are mixed. Although some studies have found that higher levels of intrusiveness among Black /African American caregivers are associated with more negative child outcomes (Clincy \& Mills-Koonce, 2013; Gueron-Sela, Bedford, et al., 2018), others have not found evidence to support this association (Diemer et al., 2021; Ispa et al., 2004). Leading hypotheses suggest that this may be due to the potential for warmth to buffer the effects of intrusiveness (Ispa et al., 2004) or that intrusiveness is a potential marker of increased caregiver monitoring related to safety concerns experienced disproportionately among racial and ethnic minorities (for review, see Tamis-LeMonda et al., 2008). Aspects of identity or cultural context may influence associations between caregiver behaviors and child development (Diemer, Treviño, Gerstein, 2021), and examining how context, including caregiver goals, may predict caregiving behaviors is relevant for research on children and families.

A small number of research groups have investigated caregiver cognitions related to achievement as antecedents of overly controlling caregiving behavior (Mageau et al., 2016). In studies focusing on the preschool period, caregivers who receive instructions that create "high pressure" for child learning (i.e., their child will be tested) engage in more controlling behavior during a homework-like task than do caregivers who receive "low pressure" instructions (Grolnick, Gurland, DeCourcey, \& Jacob, 2002). Relatedly, when reminiscing with their child, caregivers who are told that their child's memory will later be tested are more adult-centered in their conversations with their child than are caregivers who are told that their child will later be asked about their perspective (Cleveland, Reese, \& Grolnick, 2007). Overly controlling caregiving behavior may be particularly inappropriate during infancy when, beyond meeting typical developmental milestones, the child's need to perform in a specific way or to achieve a particular outcome is not especially important. In fact, Gopnik (2020) theorizes that the relatively protracted human childhood helps to resolve trade-offs between exploration (i.e., learning about 
a new environment for its own sake) and exploitation (i.e., acquiring the right knowledge to succeed in a particular environment) by providing a period of safety during which individuals can observe, experiment, and discover. Although no study has yet examined how altering caregivers' goals for interacting with their child affects intrusive caregiving in infancy, we posit that when caregivers' goals focus on ensuring infant learning, they are more likely to engage in adult-centered/intrusive caregiving behavior.

In this preregistered study, we examined whether manipulating parents' goals related to child learning when playing with their infants influenced the extent to which they engaged in intrusive caregiving behavior. Biological mothers and their 5- to 8-month-old infants participated in a ten-minute "free play" interaction in our laboratory, which was coded in two-minute epochs for the nature of maternal caregiving behavior. To direct parents' goals for the free play interaction, we randomly assigned mother-infant dyads to one of two groups. Specifically, prior to the final two minutes of the interaction, we told mothers assigned to the "teach" group to focus on teaching something to their infants for the remainder of the interaction; in contrast, we told mothers assigned to the "learn" group to focus on learning something from their infants for the remainder of the play period. In addition, we included a "control" group of mothers who received no instructions about the goals of the interaction. Overall, the present study used an experimental approach to examine changes in caregiver behavior as a result of directing caregivers' goals related to child learning. Although changes in the caregiver are likely to influence child behavior (and vice versa), we focus our analyses and interpretation on caregiver behavior as the primary target of this experimental manipulation.

Our hypotheses focused on within-person changes in mothers' behaviors across the last two epochs of the free play interaction (i.e., from the two minutes before receiving instructions [time 1 (T1)] to the two minutes after receiving instructions [time 2 (T2)]) as a function of group assignment. Overall, we hypothesized that the presence and type of mothers' explicit goals for interacting with their infant would affect the extent to which they engaged in intrusive caregiving. 
First, we hypothesized that instructing mothers to teach their infant would increase intrusiveness, such that mothers would be more intrusive after receiving these instructions than they were before receiving these instructions. Support for this hypothesis would indicate that an explicit goal to teach increases mothers' tendency to engage in behavior that interferes with the infant's exploration and autonomy. Second, we hypothesized that instructing mothers to learn from their infant would reduce intrusiveness, such that mothers would be less intrusive after these instructions than they were before these instructions. Finally, we hypothesized that mothers in the teach and learn groups would exhibit greater absolute change in intrusiveness across the last two epochs of the free play than would mothers in the control group who received no instructions about goals of the interaction. As a supplementary goal, we also collected qualitative data to explore mothers' understanding of their goals for interacting with their infant. After the interaction ended, we asked, "What was your goal in the last two minutes of this interaction?" This question was primarily used to ensure that all mothers included in the quantitative analysis comprehended the instructions; however, we also include these responses as supplementary information as they are relevant to understanding how mothers perceived their goals for interacting.

In addition to testing these hypotheses, we conducted exploratory analyses on other dimensions of caregiving. Specifically, we explored whether manipulating parents' goals related to infant learning was associated with changes in parental sensitivity, warmth/positive regard, and cognitive stimulation. Briefly, caregiver sensitivity is defined as awareness of infant cues that indicate needs, emotions, interests, and capabilities, and contingent and appropriate responses to these cues (Kok et al., 2013; Raby, Roisman, Fraley, \& Simpson, 2015). Higher levels of caregiver sensitivity have been linked to higher infant cognitive abilities, as indexed across memory, problem solving, early number concepts, generalization, classification, vocalizations, language, and social skills (Roger Mills-Koonce et al., 2015). Caregiver warmth is defined as expressions of positive feelings toward the infant, commonly demonstrated as 
smiling and using a warm tone of voice (Anderson, Goulter, \& McMahon, 2021; Daniel, Madigan, \& Jenkins, 2016). Higher levels of caregiver warmth have been associated with higher child perceptual and verbal skills (Hubbs-Tait et al., 2002). Cognitive stimulation measures the degree to which the caregiver tries to foster the infant's cognitive development through behaviors such as describing objects, focusing the infant's attention, verbally expanding on infant vocalizations, and encouraging new activities for the infant (Crosnoe et al., 2010; Matijasevich et al., 2020). Higher levels of caregiver cognitive stimulation have generally been linked with higher cognitive skills in children (Cabrera et al., 2020; Tucker-Drob \& Harden, 2012). Although different dimensions of caregiving are correlated, previous investigations indicate unique prospective associations of different types of behavior with early childhood outcomes, such as verbal and nonverbal abilities as toddlers (Hubbs-Tait et al., 2002).

\section{Design}

\section{Method}

We used a 3 (control vs. teach vs. learn group) $\times 2$ (T1 vs. T2) mixed-effects design Dyads in the control group were randomly selected from a larger sample of dyads who received no instructions about the goal of the interaction. Remaining dyads were randomly assigned to the teach and learn groups using a block randomization procedure (Suresh, 2011).

\section{Transparency and Openness}

We report how we determined our sample size, all data exclusions, all manipulations in the study. Data were analyzed using R. Data and code are available at https://github.com/lucysking/teach_learn. Caregiver coding manual is available at https://osf.io/2gp6b/?view_only=4381f7d2a4cf40b1abcf0b41f76cdf63. The study was preregistered at https://osf.io/dt7ck/?view_only=d03597cc2ab4495ba81b748756d823a9.

\section{Participants}

Participants were women and their infants who were recruited from communities in the San Francisco Bay Area to participate in the Brain and Behavior Infant Experiences (BABIES) 
project (Humphreys, King, Choi, \& Gotlib, 2018), an observational study of the association between perinatal experiences and infant psychobiological development. Mothers were on average 33.34 years old (4.44 SD); in terms of race, $58 \%$ identified as White, $27 \%$ as Asian/Asian American, 2\% as Black/African American, 3\% as Native Hawaiian/Pacific Islander, and $11 \%$ as another race; $12 \%$ identified as Latine ethnicity. Infants (48\% female sex) were on average 6.13 months old ( 0.39 SD) and in terms of race, $47 \%$ identified as White, $20 \%$ as Asian/Asian American, 1.3\% as Black/African American, and 20\% as another race, per mother report; $17.3 \%$ identified as Latine ethnicity per mother report. The sample for the current analyses included mother-child dyads who participated in a laboratory-based "free play" interaction at infant age 5-8 months. Of the 155 dyads who participated in the BABIES project at infant age 5-8 months, 142 participated in the free play interaction. Of these participants, 89 dyads were eligible for assignment to the control group. Control group participants were recruited into the study prior to preregistration of the experimental conditions; these participants did not receive instructions about goals during the final 2 minutes of the free play interaction. The remaining 53 dyads completed the free play following the preregistration and were eligible for assignment to either the teach or learn groups.

In addition to meeting inclusion/exclusion criteria for the broader study (see Procedure), criteria for inclusion in the current analyses were that mothers accurately understood the instructions about the goals of the interaction (i.e., if assigned to either the teach or learn group, responded appropriately to the post-interaction question, "What was your goal in the last two minutes of this interaction?") and that the infant did not display significant distress in the last two epochs of the interaction. Dyads with distressed infants were excluded given mothers' difficulties in attending to or following instructions while soothing their infant. Of the 89 dyads eligible for assignment to the control group, 27 were excluded because the infant displayed distress during the interaction. Distress was determined through objective coding of infant behavior and was quantified as receiving a score $\geq 2.5$ (i.e., between "low" and "moderately low") 
on the infant negative mood subscale (see Supplementary Material) of the Parent-Child Interaction Rating Scales-Infant Adaption (Bosquet Enslow, Carter, Hails, King, \& Cabrera, 2014; Sosinsky et al., 2004). From the remaining 62 dyads, we randomly selected 22 dyads to comprise the final control group. Of the 53 dyads eligible for assignment to either the teach or learn groups, 9 were excluded either because the mother did not understand the instructions based the post-interaction follow-up question about their goal for the interaction $(n=1$ in learn group) or because the infant was distressed ( $n=5$ in teach group; $n=3$ in learn group), 23 were assigned to the teach group, and 21 were assigned to the learn group. Thus, the final total sample size for the current analyses was 66 dyads.

\section{Sample Size Rationale}

The sample size for the current analyses was based on a preregistered power analysis conducted in G*Power (Faul, Erdfelder, Lang, \& Buchner, 2007) using an $\alpha=.05,80 \%$ required power, and effect sizes and variances estimated using simulated data. We found that we required a minimum total sample size of 60 (20 in each group) to obtain significant results for all planned analyses, including an omnibus interaction of the between-subjects factor of group assignment (i.e., control vs. teach vs. learn) and the within-subjects factor of time (i.e., T1 vs. T2), and each of the simple effects for this interaction (see Statistical Analyses).

\section{Procedure}

The BABIES Project was approved by the Stanford Institutional Review Board (Protocol 36366). Mothers provided informed written consent for themselves and their infants and were compensated for their time. Participants included in the current analyses were recruited either during their pregnancies (16-35 weeks gestation) or when their infants were age 6 months or younger through online advertisements and flyers posted in the local community. Participants recruited during pregnancy participated in additional sessions not included in the current analyses. All participants were screened for inclusion/exclusion criteria through a phone interview. When infants were approaching age 6 months, mother-infant dyads were invited to 
attend a laboratory session in which dyads participated in two laboratory-based, video-recorded mother-infant interactions and mothers completed questionnaires and interviews. Inclusion criteria for this session were that mothers had a singleton infant between ages $\geq 5$ and $<9$ months, were age $\geq 18$ years, were fluent in English, and had no immediate plans to leave the geographic area. Of note, English fluency was required due to lab staffing (i.e., trained staff were not available to conduct assessments or interviews in other languages). Notably, mothers were not required to speak English as a first language or to their child. Exclusion criteria included maternal bipolar disorder, maternal psychosis, maternal severe learning disabilities, severe complications during birth, infant head trauma, infant premature birth $(<36$ weeks gestation), infant congenital/genetic/neurological disorders, and contraindication for infant magnetic resonance imaging.

\section{Measures}

\section{Maternal Caregiving Behavior}

All dyads completed a ten-minute videorecorded "free play" interaction in the laboratory in which they were invited to sit on a playmat on the floor with access to a box of ageappropriate toys and were instructed to play as they usually would at home (infants often reclined against their caregiver, lay on the mat, or sat unassisted if able). Using the (Bosquet Enslow et al., 2014; Sosinsky et al., 2004), trained independent coders subsequently observed the video recordings to rate maternal intrusiveness during each 2-minute interval of the interaction. Prior to coding, we edited the videos to remove the portion in which mothers received our instructions about their goal for the interaction to ensure that coders were masked to group assignment. Possible scores for intrusiveness ranged from 1 (not at all characteristic) to 7 (very characteristic), increasing in half-point increments. The PCIRS-IA defines intrusiveness as overcontrolling, adult-centered interaction, involving behaviors characterized by the parent imposing their agenda on the infant, and is operationalized as not allowing the infant 
a "turn" or an opportunity to respond at their own pace (e.g., interrupting infant's exploration; insisting on playing with specific toys; rapid/overwhelming presentation of different stimuli; excessive/forceful physical play or touch). This conceptualization of intrusiveness is consistent with other coding systems for older ages (e.g., "adult centered and overcontrolling, imposing their own agenda on the child" from the Young Family Interaction Coding System; Paley, Cox, \& Kanoy, 2001). Moreover, the PCIRS-IA rates behaviors based on intensity and frequency, such that caregivers may receive higher scores in any 2-minute period by exhibiting behaviors that are either very intense, very frequent, or a combination of the two. Based on a randomly selected subset of 28 free play videos rated by two coders, reliability between coders for raw values of maternal intrusiveness at the level of 2-minute interval was good (ICC=.76).

Although preregistered hypotheses focused on maternal intrusiveness, coders also rated maternal sensitivity (i.e., awareness of infant's cues and contingent and appropriate responses to these cues; $I C C=.81$ ), cognitive stimulation (i.e., fostering of cognitive development in a manner that is matched to the infant's developmental level or interest; $I C C=.84$ ), positive regard/warmth (expressed positive feelings for the infant; $I C C=.85$ ), negative regard (expressed negative feelings for the infant; ICC=.76), and detachment/disengagement (lack of emotional involvement or awareness of infant's needs; ICC=.89). The PCIRS-IA was used to guide raters for all caregiving constructs assessed.

\section{Interaction Goals Induction}

For dyads assigned to the teach and learn groups, the experimenter re-entered the room when eight minutes of the free play interaction had elapsed and delivered instructions about the goals of the final two minutes of the interaction.

“Teach" Instructions. The experimenter gave the following instructions to mothers assigned to the teach group: "You are almost finished with this play period. For the next two minutes, we want you to focus on teaching your child something. Infants can learn many things from their parents, and you can choose to teach your child anything. For some people, that 
might mean teaching them about the toys in the room or other things like counting or letters. Do you understand the instructions?"

“Learn” Instructions. The experimenter gave the following instructions to mothers assigned to the teach group: "You are almost finished with this play period. For the next two minutes, we want you to focus on learning something from your child. Parents can learn many things from their infants, and your child may teach you anything. From some children, we learn about their interests in specific toys or how they are mastering new motor skills. Do you understand the instructions?"

If mothers responded that they did not understand the instructions, the experimenter rephrased the prompt, highlighting the goal to either teach or learn from the child. Following the end of the interaction, mothers were asked, "What was your goal in the last two minutes of this interaction?" If mothers responded in a manner that was not congruent with the instruction, they were excluded from the final sample.

\section{Infant, Maternal, and Family Characteristics}

To assess infant temperament, mothers completed the negative affectivity subscale (Cronbach's $\alpha=.79$ ) of the Infant Behavior Questionnaire-Revised Short Form (IBQ-R-SF; Putnam, Helbig, Gartstein, Rothbart, \& Leerkes, 2014). Mothers reported their education level, their annual household income in bins ranging from $1(\$ 0-50,000)$ to $7(>\$ 150,000)$, and the number of adults and children in their household. We calculated family income-to-needs ratio by dividing the annual household income (median point of each bin) by the county-specific lowincome threshold for the number of people in the household. Infant status as the only child in the household was determined based on whether the mother reported only one child in the household. Mothers self-reported their race and ethnicity. To assess maternal depressive symptoms, mothers completed the Center for Epidemiological Studies Depression Scale (CESD; Radloff, 1977). The 20-item CES-D instructs mothers to consider their past week and respond to items on a 4-point scale from 0 (rarely or none of the time) to 3 (most or all of the 
time), with higher scores representing greater symptoms of depression $(\alpha=.88)$. To assess maternal recent life stress, mothers completed the Crisis in Family Systems-Revised (CRISYS; Berry, Quinn, Shalowitz, \& Wolf, 2001). The CRISYS is a 72-item checklist of life stressors occurring across the previous six months, including potentially traumatic events (e.g., death of a loved one), threats in the environment (e.g., hearing gunshots in neighborhood), financial problems (e.g., missing a rent or mortgage payment), family conflict (e.g., disagreements with partner), and discrimination (e.g., unfair treatment due to race or sex).

\section{Statistical Analyses}

To examine whether the three groups differed in demographic, infant temperament, caregiver life experience or mental health characteristics, we conducted chi-square or Analysis of Variance (ANOVA) tests. To test the hypothesis that intrusiveness depends on both group (between-subjects: control vs. teach vs. learn group) and time (within-subjects: T1 vs. T2), we conducted a mixed-effects ANOVA with an interaction between these two variables. We implemented the mixed-effects ANOVA using the "Ime4" package in R (Bates, Machler, Bolker, \& Walker, 2014), modeling a group (dummy-coded) $\times$ time (dummy-coded) interaction and a random effect of participant intercept. In the presence of a significant omnibus group $\times$ time interaction, we examined the following simple effects. First, we tested the hypotheses that intrusiveness increases in the teach group from T1 to T2 and that intrusiveness decreases in the learn group from T1 to T2. Second, we tested the hypotheses that increases in intrusiveness from T1 to T2 are greater in the teach group than in the control group and that decreases in intrusiveness are greater in the learn group than in the control group.

\section{Results}

We present descriptive statistics for the study sample in Table 1. We present distributions for continuous covariates and caregiving behaviors in the Supplementary Materials. Dyads in the control, teach, and learn groups did not differ significantly with respect to infant 
age, infant sex, whether the infant was an only child, infant temperamental negative affectivity, family income-to-needs ratio, maternal age, maternal depressive symptoms, maternal recent life stress, maternal education, or maternal race/ethnicity.

Table 1. Study sample characteristics.

\begin{tabular}{|c|c|c|c|c|}
\hline \multirow[b]{2}{*}{ Variable } & \multicolumn{3}{|c|}{ Mean (SD) or $\mathbf{N}$} & \multirow[b]{2}{*}{$F$ or $\chi^{2}$} \\
\hline & $\begin{array}{c}\text { Control } \\
(n=22)\end{array}$ & $\begin{array}{l}\text { Teach } \\
(n=21)\end{array}$ & $\begin{array}{l}\text { Learn } \\
(n=23)\end{array}$ & \\
\hline Infant age & $6.11(0.51)$ & $6.06(0.28)$ & $6.21(0.38)$ & $0.75, p=.475$ \\
\hline Maternal age & $35.20(4.12)$ & $32.62(3.59)$ & $32.23(5.53)$ & $2.82, p=.067$ \\
\hline Income-to-needs ratio & $1.52(0.46)$ & $1.54(0.47)$ & $1.49(0.55)$ & $0.06, p=.945$ \\
\hline $\begin{array}{l}\text { Maternal depressive } \\
\text { symptoms }\end{array}$ & $9.32(7.45)$ & $8.38(7.45)$ & $12.48(9.89)$ & $1.46, p=.239$ \\
\hline $\begin{array}{l}\text { Maternal recent life } \\
\text { stress }\end{array}$ & $5.45(3.34)$ & $5.65(3.59)$ & $7.1(6.39)$ & $0.75, p=.475$ \\
\hline Infant negative affectivity & $3.17(0.66)$ & $3.03(0.72)$ & $3.26(0.78)$ & $0.53, p=.591$ \\
\hline Infant female sex & 9 & 11 & 12 & $0.76, p=.684$ \\
\hline Infant only child & 13 & 10 & 10 & $1.16, p=.558$ \\
\hline $\begin{array}{l}\text { Maternal education } \geq \\
4 \text {-year college degree }{ }^{1}\end{array}$ & 21 & 19 & 17 & \\
\hline Maternal race ${ }^{2}$ & & & & $0.56, p=.756$ \\
\hline White & 12 & 12 & 14 & \\
\hline Asian/Asian American & 6 & 8 & 4 & \\
\hline Black/African American & 0 & 0 & 1 & \\
\hline $\begin{array}{r}\text { Native Hawaiian/Other } \\
\text { Pacific Islander }\end{array}$ & 1 & 0 & 1 & \\
\hline Another race & 3 & 1 & 3 & \\
\hline Maternal Latine ethnicity ${ }^{1}$ & 3 & 2 & 3 & \\
\hline Infant race ${ }^{2}$ & & & & $2.07, p=.356$ \\
\hline White & 11 & 10 & 14 & \\
\hline Asian/Asian American & 5 & 8 & 2 & \\
\hline Black/African American & 0 & 0 & 1 & \\
\hline $\begin{array}{r}\text { Native Hawaiian/Other } \\
\text { Pacific Islander }\end{array}$ & 0 & 0 & 0 & \\
\hline Another race & 6 & 3 & 6 & \\
\hline Infant Latine ethnicity ${ }^{1}$ & 6 & 2 & 5 & \\
\hline
\end{tabular}

Note. $N=66$ mother-infant dyads total. ${ }^{1}$ Chi-square not computed given cell sizes $<5 .{ }^{2} \mathrm{Chi}-$ square test comparing the number of mothers or infants who identified as persons of color, Latine, or another race vs. mothers who identified as White and not Latine. Maternal depressive symptoms assessed by the CES-D; Maternal recent life stress assessed by the CRISYS; Infant negative affectivity assessed by the IBQ-R-SF. 


\section{Effects of Inducing Interaction Goals on Maternal Intrusiveness}

Supporting our hypothesis, we found that change in maternal intrusiveness from T1 to T2 depended on group assignment (i.e., the presence and type of the explicit goals for the interaction; group $\times$ time: $F(2,63)=5.52, p=.006, \eta_{p}{ }^{2}=.15,95 \% \mathrm{CI}[0.03,0.28]$; see Figure 1).

Results of simple effects analyses partially supported our hypotheses. As we hypothesized, mothers who received instructions to teach their infant were more intrusive after receiving these instructions than they were before receiving these instructions $(\beta=0.60,95 \% \mathrm{Cl}[0.24,0.96]$, $\mathrm{B}=0.69, \mathrm{SE}=0.21, t(63)=3.24, p=.002 ;$ mean[SD] at $\mathrm{T} 1=2.43[1.18]$ vs. at $\mathrm{T} 2=3.12[1.37])$. Further, changes in intrusiveness following instructions to learn from the infant was not statistically significant $(\beta=-0.25,95 \% \mathrm{Cl}[-0.59,0.10], \mathrm{B}=-0.28, \mathrm{SE}=0.20, t(63)=-1.39, p=.170 ;$ mean[SD] at $\mathrm{T} 1=2.41[0.96]$ vs. at $\mathrm{T} 2=2.13[1.01])$. Mothers in the control group did not change in intrusiveness from T1 to T2 (i.e., the standardized mean difference was close to zero; $\beta=0.08$, $95 \% \mathrm{Cl}[-0.28,0.43], \mathrm{B}=0.09, \mathrm{SE}=0.35, t(63)=0.44, p=.664$; mean[SD] at $\mathrm{T} 1=2.80[1.26] \mathrm{vs}$. at $\mathrm{T} 2=2.57[1.47])$. On average, the change in intrusiveness from T1 to T2 among mothers in the teach group was larger than the change observed in the control group $(\beta=0.52,95 \% \mathrm{Cl}[0.01$, 1.03], $t(63)=2.01, \mathrm{~B}=0.60, \mathrm{SE}=0.30, p=.048)$; however, change in intrusiveness among mothers in the learn group was not significantly different from that observed in the control group $(\beta=-$ $0.32,95 \% \mathrm{Cl}[-0.82,0.17], \mathrm{B}=-0.37, \mathrm{SE}=0.20, t(63)=-1.28, p=.204)$.

These findings were identical when including the preregistered covariates of birth order (only child in household [i.e., eldest] vs. not only child]) and the sex of the infant. There were no main effects of birth order or infant sex on maternal intrusiveness ( $p$-values $>.40, \beta$-values $<$ 0.19). Our findings were also similar when, instead of comparing intrusiveness at T2 to intrusiveness in the two minutes prior to the induction, we compared intrusiveness at T2 to intrusiveness across the full eight minutes of the free play period prior to the goal induction (see 
Supplementary Material). Thus, the effect of the goal induction did not depend on treating the two minutes prior to the induction as the baseline level of intrusiveness.

\section{Effects of Inducing Interaction Goals on Other Caregiving Behaviors}

We explored whether the presence and type of explicit goals for the interaction influenced additional aspects of maternal caregiving behavior. We did not find evidence that group assignment influenced changes in maternal sensitivity, detachment, and negative regard. However, as depicted in Figure 2, we found changes in maternal cognitive stimulation and maternal warmth from T1 to T2 as a function of group assignment (stimulation: group $\times$ time $F(2,60.79)=13.24, p<.001, \eta_{p}^{2}=.30,95 \% \mathrm{Cl}[0.14,0.44]$; warmth: group $\times$ time $F(2,63)=3.44$, $\left.p=.038, \eta_{p}^{2}=.10,95 \% \mathrm{CI}[<0.01,0.21]\right)$. We describe these effects in detail below.

Whereas mothers in both the control and learn groups were less cognitively stimulating at T2 than at T1 (control: $\beta=-0.41,95 \% \mathrm{Cl}[-0.82,-0.01], \mathrm{B}=-0.34, \mathrm{SE}=0.17, t(60.08)=-1.96$, $p=.054 ; \operatorname{mean}[\mathrm{SD}]$ at T1 $=3.27[0.88]$ vs. at T2=2.98[1.06]; learn: $\beta=-0.51,95 \% \mathrm{Cl}[-0.92,-0.11]$, $\mathrm{B}=-0.42, \mathrm{SE}=0.17, t(62.27)=-2.49, p=.016 ; \operatorname{mean}[\mathrm{SD}]$ at $\mathrm{T} 1=3.00[0.64]$ vs. at $\mathrm{T} 2=2.61[0.69])$, mothers in the teach group became more cognitively stimulating across timepoints $(\beta=0.85$, $95 \% \mathrm{Cl}[0.44,1.26], \mathrm{B}=0.69, \mathrm{SE}=0.17, t(60.08)=4.06, p<.001 ;$ mean[SD] at $\mathrm{T} 1=2.90[0.54]$ vs. at T2=3.60[0.72]), suggesting that inducing the goal to teach something to the infant counteracted an otherwise typical deterioration in cognitive stimulation from earlier to later in the free play interaction. Changes in cognitive stimulation among mothers in the learn group were not significantly different from those observed in the control group $(\beta=0.10,95 \% \mathrm{Cl}[-0.47,0.68]$, $\mathrm{B}=0.08, \mathrm{SE}=0.24, t(61.15)=0.35, p=.725)$. In contrast, the increase in cognitive stimulation observed among mothers in the teach group was significantly stronger than the change in stimulation observed in the control group $(\beta=-1.36,95 \% \mathrm{Cl}[-1.84,-0.68], \mathrm{B}=-1.11, \mathrm{SE}=0.24$, $t(61.15)=-4.26, p<.001)$.

Whereas mothers in the control and teach groups did not change significantly in warmth from T1 to T2 (control: $\beta=0.12,95 \% \mathrm{Cl}[-0.23,0.48], \mathrm{B}=0.11, \mathrm{SE}=0.17, t(63)=0.68, p=.499$; 
mean[SD] at T1=4.84[0.97] vs. at T2=4.65[1.05]; teach: $\beta=-0.05,95 \% \mathrm{Cl}[-0.42,0.31], \mathrm{B}=-0.05$, $\mathrm{SE}=0.17, t(63)=-0.28, p=.781$; mean[SD] at $\mathrm{T} 1=4.00[0.87]$ vs. at $\mathrm{T} 2=3.95[0.91])$, mothers in the learn group were significantly less warm at T2 than they were at T1 $(\beta=-0.52,95 \% \mathrm{Cl}[-0.87,-$ $0.17], \mathrm{B}=-0.48, \mathrm{SE}=0.16, t(63)=-2.93, p=.005 ; \operatorname{mean}[\mathrm{SD}]$ at $\mathrm{T} 1=4.24[0.88]$ vs. at $\mathrm{T} 2=3.76[0.60])$, suggesting that inducing the goal of learning something from the infant dampened the expression of positive feelings toward the infant. The decrease in maternal warmth among mothers in the learn group was significantly more negative than that observed among mothers in the control group $(\beta=0.65,95 \% \mathrm{Cl}[0.15,1.15], \mathrm{B}=0.59, \mathrm{SE}=0.23, t(128)=-2.53, p=.014)$ and was larger in absolute value, but not significantly different, than the change observed among mothers in the teach group $(\beta=0.47,95 \% \mathrm{Cl}[-0.040 .98], \mathrm{B}=0.43, \mathrm{SE}=0.24, t(128)=-1.83$, $p=.073){ }^{1}$

Given evidence that inducing interaction goals influenced the extent to which mothers engaged in cognitively stimulating and warm behaviors, we conducted additional analyses in which we tested our preregistered hypotheses when adjusting for both mean and time-varying (person-mean-centered) levels of cognitive stimulation and warmth. Our findings were highly similar, and we did not find significant main effects of mean or time-varying levels of cognitive stimulation or warmth on intrusiveness, suggesting that maternal intrusiveness was not coupled with changes in maternal cognitive stimulation or warmth. In other words, despite group-level increases in both intrusiveness and cognitive stimulation among mothers who were directed to teach their infant, and group-level decreases in both intrusiveness and warmth among mothers who were directed to learn from their infant, changes in intrusiveness and these other caregiving behaviors were not associated across time, indicating that there are largely distinct effects of the

\footnotetext{
${ }^{1}$ We did not anticipate that the timescale infant behavior or development changes in response to the parent goals manipulation to be immediate. However, we did explore whether the experimental manipulation of parent goals had an immediate effect on infant mood in the remaining 2 minutes of the interaction. See Supplementary Materials.
} 
goal induction on different aspects of caregiving behavior. Further, results with respect to the effects of group and time on maternal intrusiveness were highly similar when adjusting for these other caregiving behaviors (see Supplementary Materials).

\section{What Were Mothers' Perceived Goals and What Did They Learn?}

We also collected qualitative data to allow us to explore mothers' understanding and perception of their goals for interacting with their infant. After the interaction ended, we asked, "What was your goal in the last two minutes of this interaction?" Responses to this question are listed in full in the Supplementary Materials. Mothers in the teach condition typically described teaching general skills or specific tasks. For example, they responded that their goal was to teach the infant, "to identify things; we looked at colors, then body parts," "to get him familiarized with animals," "to show her that you can put objects on top of each other," and to "have him pay attention when l'm saying colors." Others responded, referring to the toys we provided, that their goal was "to teach her to push the yellow button," "to get him to push the button," "to teach her how to play with a toy that pops out," and "to put the toys in the box."

Mothers in the learn condition typically described observing their infant in order to understand their abilities and interests, and watching their infant explore. For example, mothers responded that they "focused on what she was trying to communicate," "wanted to see whether he would be able to make a choice between toys," "wanted to see if he might stack the blocks or hold two blocks at once," and "to figure out which toy she liked." Others said their goal was "to watch her play and think about...the new skills that she's learning," and to "figure out something new from the toys she was playing with and let her guide the play." When we asked these mothers specifically what they learned from their baby, many said they learned about previously unnoticed infant capacities. For example, they said "she was able to frown to indicate she didn't want [the toy]—she's learning to frown and look disappointed," "she doesn't need me to entertain her as much as I think she does—she's capable of exploring things on her own," "he can lean very far forward and pick something up very intentionally with two hands, and he's not 
mimicking what I'm doing, he's just on his own," and "that she was able to sit without any support, and that she was able to adjust with a new environment."

Overall, these qualitative responses suggest that mothers in the learn condition noticed new things about their infants through a process of observing infant-led play; some mothers reported discovering in those two minutes that their infant was more autonomous than they realized. Not surprisingly, mothers in the teach condition tended to implement a lesson plan for their infant and focused on engaging the infant with this agenda.

Figure 1. Changes in maternal intrusiveness depend on the presence and type of the explicit goal for the interaction.

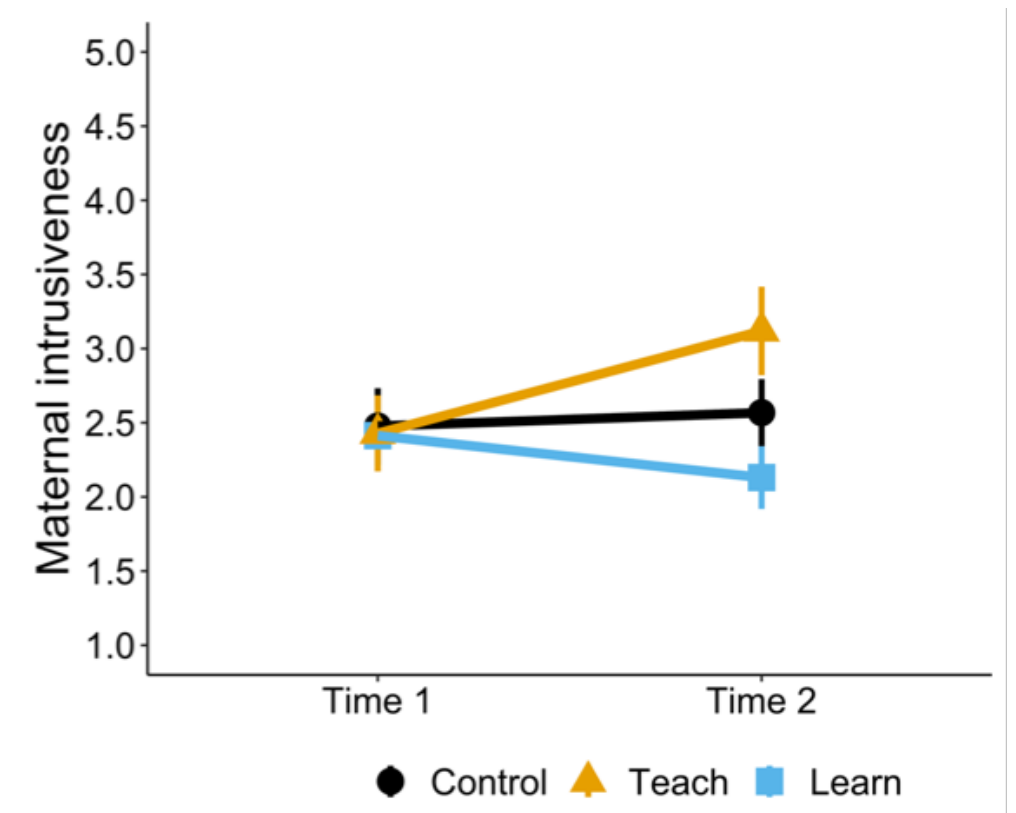

Note. Time 1=two minutes before receiving instructions. Time 2=two minutes after receiving instructions. Points are means and point ranges are standard errors. Mothers in the control group received no instructions; mothers in teach group received instructions to teach their infant something; mothers in the learn group received instructions to learn something from their infant. 
Figure 2. Inducing interaction goals also changes levels of $(A)$ cognitive stimulation and (B) positive regard.

A

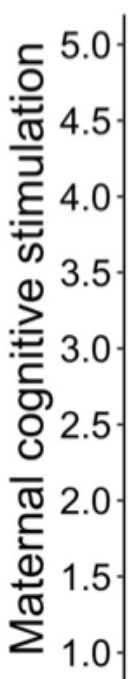

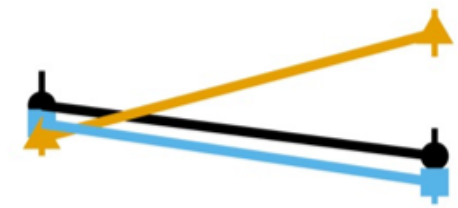

Time 1

Time 2

- Control 4 Teach Learn

B

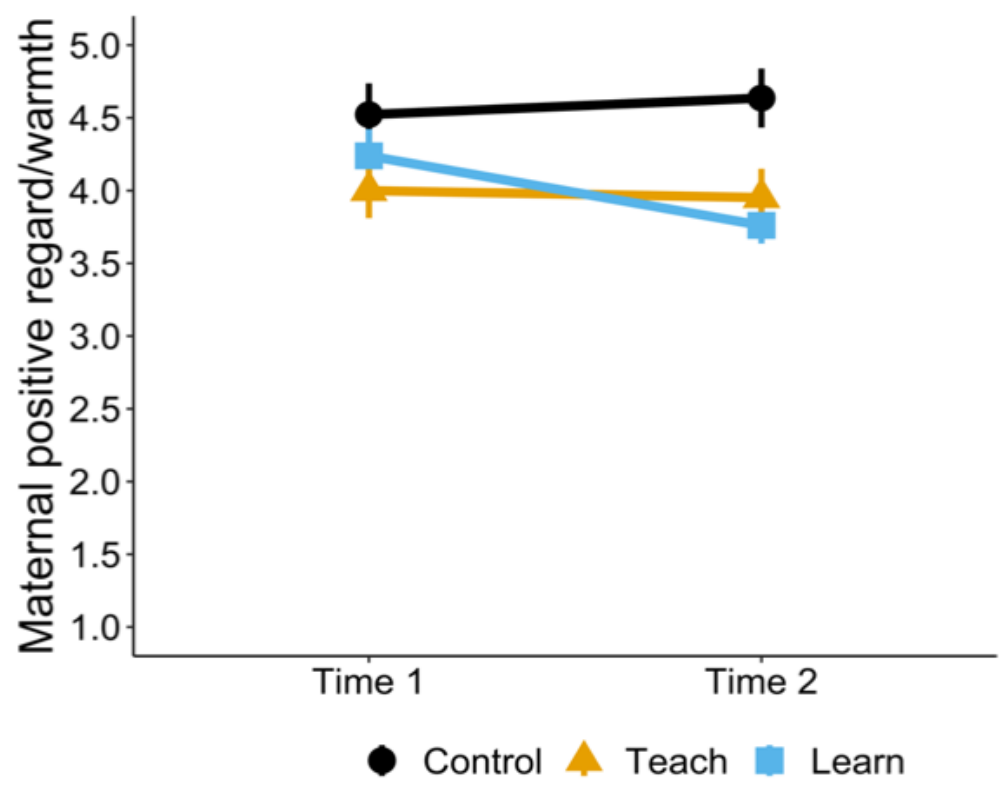

Note. Time $1=$ two minutes before receiving instructions. Time 2=two minutes after receiving instructions. Points are means and point ranges are standard errors. Panel A: cognitive stimulation. Panel B: positive regard/warmth. 


\section{Discussion}

In this study, we randomly assigned mothers to receive different instructions regarding their goals for interacting with their 6-month-old infants and observed how much they engaged in intrusive caregiving behavior. Consistent with our pre-registered hypotheses, we found that manipulating parents' explicit goals regarding infant learning influenced the degree to which they exhibited intrusive caregiving behaviors. When mothers received instructions to focus on teaching something to their infant, they significantly increased in intrusiveness. This increase constituted a medium to large effect size, and an approximate 1-point increase in the observed score for intrusiveness on a scale ranging from 1 (very low; no signs of intrusive behavior) to 7 (very high; interactions are typically and consistently intrusive). In contrast, when mothers received instructions to focus on learning something from their infant, they subsequently decreased in intrusiveness, although not significantly so, and mothers in a control group who received no instructions maintained the same level of intrusiveness across time.

These findings suggest that caregivers' goals for child learning can lead to overly controlling and adult-centered caregiving behavior. Grolnick (2003) proposed that when caregivers perceive pressure regarding their child's achievement, they adopt outcome-oriented goals at the cost of their child's autonomy. Supporting this formulation, researchers have found that when caregivers of older children and adolescents have goals that emphasize their child's performance, they engage in more controlling behavior (Cleveland et al., 2007; Gonida \& Cortina, 2014; Grolnick et al., 2002; Mageau et al., 2016). The current study extends these findings to infancy, a developmental period in which caregivers' goals focused on child achievement are especially out of step with the need for child-led exploration. To seat these findings within the broader literature, it is important to note that the capacity of infants and young children to learn about their environments through exploration and play is in tension with adults' tendency to solve problems by exploiting what they already know (Gopnik, 2020). In fact, young children outperform older children and adults on tasks that require cognitive flexibility (Gopnik et 
al., 2017). Infants may learn better through exploration than through formal teaching because they use surprising and unusual events as cues for engaging in more information-seeking (Stahl \& Feigenson, 2015). They may also better adapt to novel information when their caregivers do not insist that they engage in this information in a particular way. For example, toddlers whose mothers make fewer demands that they approach novel and potentially threatening objects have been found to cope with these objects more competently (Nachmias, Gunnar, Mangelsdorf, \& Buss, 1996). Thus, when caregivers focus on teaching their infant specific information or skills in an adult-centered manner, they may interfere with, rather than promote, their infant's development.

Findings of this study also build on previous research by indicating that simply inducing the broad goal of teaching one's infant, without applying specific pressure regarding the infant's performance, is sufficient to increase intrusive caregiving. The nature of this goal induction is important for considering how our findings may generalize outside of the laboratory. First, the present findings should be considered in context, such that the experimental manipulation of parent teaching goals increased parent intrusiveness in a relatively well-resourced sample of parents who predominantly identify as White or Asian/Asian-American from the San Francisco Bay Area of the U.S. Second, implications of these findings should be considered across multiple dimensions. Cultural orientations regarding achievement may influence caregivers' goals for interacting with their children (Prevoo \& Tamis-LeMonda, 2017; Tamis-LeMonda, Way, et al., 2008). Though in the present study we experimentally manipulated parents' goals, there is naturally-occurring variation in caregivers' goals based on a number of factors. Contemporary industrialized societies are increasingly competitive. Whereas in the past, many U.S. caregivers could expect that their child would have similar or greater success than they did, rising income inequality has contributed to fading socioeconomic mobility (Chetty et al., 2017). In the face of diminishing opportunities, caregivers may believe that preparing their child to behave and think in particular ways is important for maximizing the probability of their child's success. In this 
context, broad messaging that encourages caregivers to stimulate infant learning (e.g., "you can teach your infant many things") may actually elicit intrusive caregiving behavior, which is associated with decreased infant learning over time. Indeed, in harsh and/or unpredictable environments in which opportunities are fleeting, individuals favor exploitation over exploration, or finding the "right" information to ensure rapid success over the slower discovery of novel information (Frankenhuis, Panchanathan, \& Nettle, 2016; Humphreys et al., 2015; Kidd, Palmeri, \& Aslin, 2013), and so these messages may be compounding in the face of rising income inequality. Future research should examine how environmental conditions, whether naturally occurring or experimentally induced, moderate the effects of caregivers' goals regarding infant learning on their caregiving behavior.

Of course, there is also variation within cultures in how caregivers behave when they adopt the goal of teaching their child. In the current study, we found that, at the group level, mothers who received instructions to teach their infant subsequently provided more cognitive stimulation on average. From one perspective, this finding simply instantiates our manipulation-we told mothers to teach their infant and they followed our instructions. It is interesting, however, that increases in cognitive stimulation were not coupled with increases in intrusiveness, indicating that some mothers who were instructed to teach their infant enacted this goal without being controlling or adult centered. Indeed, of the 21 mothers in the teach group, $14(67 \%)$ increased in intrusiveness but the remaining 7 either did not change or decreased in intrusiveness. In adolescents, researchers have distinguished caregivers' performance achievement goals, which focus on demonstrations of child knowledge and skills compared to their peers, from caregivers' mastery achievement goals, which focus on the growth of competence within the context of that child's development (Mageau et al., 2016). Whereas caregivers with performance goals exert more psychological control over their adolescents, caregivers with mastery goals are less controlling (Mageau et al., 2016). Among caregivers of young children, those who focus on the process rather than the outcome of child 
learning are less intrusive (Cleveland et al., 2007; Grolnick et al., 2002). It is possible, therefore, that group level differences in both intrusiveness and cognitive stimulation were driven, in part, by outcome- or performance-oriented behaviors for the former and process- or mastery-oriented behaviors for latter.

Although we found that mothers who received instructions to learn something from their infant were subsequently less intrusive and, based on their open-ended responses, discovered new things about their infants' capabilities, these mothers were also less cognitively stimulating and less warm. Further, the group-level decrease in intrusiveness among mothers in the "learn" group was not statistically significant. Outside the laboratory, messages directed toward caregivers that value growth in infant competence over time rather than the performance of specific skills may motivate caregivers to enrich their infants' environments without undermining their infants' autonomy. Notably, however, in motivating parents to focus on what their infant thinks, does, and desires, it is important not to disrupt parents' expressions of positive emotions toward their infant. Future investigations of caregiver goals may consider an experimental manipulation focused on caregiver attuned participatory play with their infant rather than explicit learning from infant goals to support caregiver value in growth without increases in intrusiveness. For example, longer-term interventions that effectively reduce caregiver intrusiveness and increase sensitivity, such as Attachment and Biobehavioral Catch-Up, combine training in how to show delight towards one's child with following the child's lead (Dozier \& Bernard, 2017).

\section{Limitations}

This is the first study to investigate how directing parents' goals regarding infant learning influences intrusive caregiving behavior, and its limitations highlight avenues for future research. First, the increase in intrusive caregiving behavior observed when mothers were in the teaching condition is notable. Such behaviors may impact infant development when they occur with high frequency; however, such an impact on child behavior and development is theorized to unfold 
over time and is not something necessarily observable in response to a brief experiment (e.g., Grumi, Pettenati, Manfredini, \& Provenzi, 2022) and thus we did not focus on infant behavior change in the current investigation. Examining how caregiver goals and behaviors are associated with infant outcomes over time is necessary for determining whether and to what extent goals for teaching versus learning matter for child behavior and development. Relatedly, the brief goals manipulation produced a medium to large effect on caregiver intrusiveness in the teach condition; however, we are not able to determine what levels of intrusiveness constitute a clinical concern or if caregivers were more likely to engage in clinically concerning intrusiveness as a function of the manipulation. Second, the sample size was sufficient for the strict focus on experimental manipulation of goals, though did not provide the power necessary for exploration of moderators (e.g., cultural orientation, values, etc.). Examining the degree to which caregivers' goals to teach or learn from infants are influenced by culture, identity, and other relevant characteristics represents an important area for additional study. In addition, it is unclear to what extent our findings generalize both outside the laboratory and to other geographic regions and cultures, or to non-maternal caregivers (e.g., fathers, grandparents, childcare professionals). Although we did not communicate to mothers that we would evaluate their infants' learning, some mothers in the "teach" group may have experienced greater pressure regarding their infant's performance in the laboratory environment than they would in their naturalistic environment. Future studies should evaluate whether caregivers who naturalistically adopt goals focused on teaching their infants also engage in more intrusive caregiving. As a related point, most mothers in this study were White or Asian American and tended to be highly educated. Although we were not statistically powered to investigate variation in the impact of our experimental manipulation as a function of race, ethnicity, cultural orientation, or socioeconomic status, it is possible that mothers from different backgrounds behave differently when asked to teach or learn from their infant, especially in a laboratory context. Another limitation is that, unlike the dyads in the two groups who received the experimental manipulation, dyads in the 
control condition did not receive any interruption in the free play activity. It may be beneficial in future work to include an interruption by research staff to further reduce differences across all three conditions. The nature of our instructions to mothers, occurring after 8 minutes of a free play interaction, may have been a time when dyads were relatively fatigued.

\section{Conclusion}

The transition to parenthood brings dramatic psychosocial changes that are often challenging (Saxbe, Goldenberg, \& Rossin-Slater, 2018); many new caregivers experience stress about how best to care for their infant, including how to best support their infant's learning. Our findings suggest that explicit goals to teach infants increases caregiving intrusiveness on average, which has been demonstrated to undermine infant learning. Prompting caregivers to engage in explicit teaching may interfere with infants' autonomy and exploration. Thus, broad messages encouraging caregivers to foster infant learning may come with risks. Nonetheless, some caregivers are able to adopt the goal of teaching their infant without becoming intrusive. Future research should investigate how to motivate caregivers to provide cognitive stimulation without unintentionally also increasing adult-centered and controlling caregiver behavior. 


\section{References}

Anderson, S. L., Goulter, N., \& McMahon, R. J. (2021). Examining the directionality of the relationship between maternal warmth and early school-age anxiety. Child Psychiatry and Human Development. doi: 10.1007/s10578-021-01197-4

Bates, D., Machler, M., Bolker, B., \& Walker, S. (2014). Fitting linear mixed-effects models using Ime4. Journal of Statistical Software, 1-51. doi: 10.18637/jss.v067.i01

Bernier, A., Carlson, S. M., Deschênes, M., \& Matte-Gagné, C. (2012). Social factors in the development of early executive functioning: A closer look at the caregiving environment. Developmental Science, 15(1), 12-24. doi: 10.1111/j.1467-7687.2011.01093.x

Berry, C., Quinn, K., Shalowitz, M., \& Wolf, R. (2001). Validation of the Crisis in Family Systems-Revised, a contemporary measure of life stressors. Psychological Reports, 88(3), 713-724. doi: 10.2466/pr0.2001.88.3.713

Bosquet Enlow, M., Carter, A., Hails, K., King, L., \& Cabrera, I. (2014). Parent-Child Interaction Rating Scales - Infant Adaptation Manual.

Cabrera, N. J., Moon, U., Fagan, J., West, J., \& Aldoney, D. (2020). Cognitive Stimulation at Home and in Child Care and Children's Preacademic Skills in Two-Parent Families. Child Development, 91(5), 1709-1717. doi: 10.1111/cdev.13380

Cabrera, N. J., Shannon, J. D., \& Tamis-LeMonda, C. (2007). Fathers' influence on their children's cognitive and emotional development: From toddlers to pre-K. Applied Developmental Science, 11(4), 208-213. doi: 10.1080/10888690701762100

Chetty, R., Grusky, D., Hell, M., Hendren, N., Manduca, R., \& Narang, J. (2017). The fading American dream: Trends in absolute income mobility since 1940. Science, 406(April), 398406.

Cleveland, E. S., Reese, E., \& Grolnick, W. S. (2007). Children's engagement and competence in personal recollection: Effects of parents' reminiscing goals. Journal of Experimental Child Psychology, 96(2), 131-149. doi: 10.1016/j.jecp.2006.09.003 
Clincy, A. R., \& Mills-Koonce, W. R. (2013). Trajectories of intrusive parenting during infancy and toddlerhood as predictors of rural, low-income African American boys' school-related outcomes. American Journal of Orthopsychiatry, 83(2 PART 3), 194-206. doi:

10.1111/ajop.12028

Clincy, A. R., \& Mills-Koonce, W. R. (2013). Trajectories of intrusive parenting during infancy and toddlerhood as predictors of rural, low-income African American boys' school-related outcomes. American Journal of Orthopsychiatry, 83(2-3), 194-206. doi: 10.1111/ajop. 12028

Diemer, M. C., Treviño, M. S., \& Gerstein, E. D. (2021). Contextualizing the role of intrusive parenting in toddler behavior problems and emotion regulation: Is more always worse? Developmental Psychology, 57(8), 1242-1253. doi: 10.1037/dev0001231

Distefano, R., Galinsky, E., McClelland, M. M., Zelazo, P. D., \& Carlson, S. M. (2018). Autonomy-supportive parenting and associations with child and parent executive function. Journal of Applied Developmental Psychology, 58(October 2017), 77-85. doi: 10.1016/j.appdev.2018.04.007

Dotterer, A. M., Iruka, I. U., \& Pungello, E. (2012). Parenting, race, and socioeconomic status: Links to school readiness. Family Relations, 61(4), 657-670. doi: 10.1111/j.17413729.2012.00716.x

Dozier, M., \& Bernard, K. (2017). Attachment and Biobehavioral Catch-up: Addressing the needs of infants and toddlers exposed to inadequate or problematic caregiving. Current Opinion in Psychology, 15, 111-117. doi: 10.1016/j.copsyc.2017.03.003

Faul, F., Erdfelder, E., Lang, A.-G., \& Buchner, A. (2007). G*Power 3: A flexible statistical power analysis program for the social, behavior, and biomedical sciences. Behavior Research Methods, 39, 175-191. 
Frankenhuis, W. E., Panchanathan, K., \& Nettle, D. (2016). Cognition in harsh and unpredictable environments. Current Opinion in Psychology, 7, 76-80. doi: 10.1016/j.copsyc.2015.08.011

Gonida, E. N., \& Cortina, K. S. (2014). Parental involvement in homework: Relations with parent and student achievement-related motivational beliefs and achievement. British Journal of Educational Psychology, 84(3), 376-396. doi: 10.1111/bjep.12039

Gopnik, A. (2020). Childhood as a solution to explore-exploit tensions. Philosophical Transactions of the Royal Society B: Biological Sciences, 375(1803). doi: $10.1098 /$ rstb.2019.0502

Gopnik, A., O'Grady, S., Lucas, C. G., Griffiths, T. L., Wente, A., Bridgers, S., ... Dahl, R. E. (2017). Changes in cognitive flexibility and hypothesis search across human life history from childhood to adolescence to adulthood. Proceedings of the National Academy of Sciences of the United States of America, 114(30), 7892-7899. doi: 10.1073/pnas.1700811114

Grolnick, W. S. (2003). The psychology of parental control: How well-meant parenting backfires. Mahwah, NJ: Lawrence Erlbaum Associates.

Grolnick, W. S., Gurland, S. T., DeCourcey, W., \& Jacob, K. (2002). Antecedents and consequences of mothers' autonomy support: an experimental investigation. Developmental Psychology, 38(1), 143-155. doi: 10.1037/0012-1649.38.1.143

Grolnick, W. S., \& Pomerantz, E. M. (2009). Issues and challenges in studying parental control: Toward a new conceptualization. Child Development Perspectives, 3(3), 165-170. doi: 10.1111/j.1750-8606.2009.00099.x

Grumi, S., Pettenati, G., Manfredini, V., \& Provenzi, L. (2022). Flexibility and organization in parent-child interaction through the lens of the dynamic system approach: A systematic review of State Space Grid studies. Infant Behavior and Development, 67, 101722. doi: 10.1016/j.infbeh.2022.101722 
Gueron-Sela, N., Bedford, R., Wagner, N. J., \& Propper, C. B. (2018). Children's Executive Function Attenuate the Link Between Maternal Intrusiveness and Internalizing Behaviors at School Entry. Journal of Clinical Child \& Adolescent Psychology, 47(sup1), S435-S444. doi: $10.1080 / 15374416.2017 .1381911$

Gueron-Sela, N., Camerota, M., Willoughby, M. T., Vernon-Feagans, L., Cox, M. J., Greenberg, M. T., ... Mills-Koonce, W. R. (2018). Maternal depressive symptoms, mother-child interactions, and children's executive function. Developmental Psychology, 54(1), 71-82. doi: $10.1037 / \operatorname{dev} 0000389$

Hastings, P. D., \& Grusec, J. E. (1998). Parenting goals as organizers of responses to parentchild disagreement. Developmental Psychology, 34(3), 465-479. doi: 10.1037/00121649.34.3.465

Hembacher, E., \& Frank, M. C. (2020). The Early Parenting Attitudes Questionnaire: Measuring intuitive theories of parenting and child development. Collabra: Psychology, 6(1), 1-11. doi: 10.1525/collabra.190

Hubbs-Tait, L., Culp, A. M., Culp, R. E., \& Miller, C. E. (2002a). Relation of maternal cognitive stimulation, emotional support, and intrusive behavior during Head Start to children's Kindergarten cognitive abilities. Child Development, 73(1), 110-131. doi: 10.1111/1467$\underline{8624.00395}$

Hubbs-Tait, L., Culp, A. M., Culp, R. E., \& Miller, C. E. (2002b). Relation of Maternal Cognitive Stimulation, Emotional Support, and Intrusive Behavior during Head Start to Children's Kindergarten Cognitive Abilities. Child Development, 73(1), 110-131. doi: 10.1111/14678624.00395

Humphreys, K. L., King, L. S., Choi, P., \& Gotlib, I. H. (2018). Maternal depressive symptoms, self-focus, and caregiving behavior. Journal of Affective Disorders, 238, 465-471. doi: 10.1016/j.jad.2017.07.051 
Humphreys, K. L., Lee, S. S., Telzer, E. H., Gabard-Durnam, L. J., Goff, B., Flannery, J., \& Tottenham, N. (2015). Exploration-exploitation strategy is dependent on early experience. Developmental Psychobiology, (August). doi: 10.1002/dev.21293

Ispa, J. M., Fine, M. A., Halgunseth, L. C., Harper, S., Robinson, J. A., Boyce, L., ... BradySmith, C. (2004a). Maternal intrusiveness, maternal warmth, and mother-toddler relationship outcomes: Variations across low-income ethnic and acculturation groups. Child Development, 75(6), 1613-1631. doi: 10.1111/j.1467-8624.2004.00806.x

Ispa, J. M., Fine, M. A., Halgunseth, L. C., Harper, S., Robinson, J., Boyce, L., ... Brady-Smith, C. (2004b). Maternal Intrusiveness, Maternal Warmth, and Mother-Toddler Relationship Outcomes: Variations Across Low-Income Ethnic and Acculturation Groups. Child Development, 75(6), 1613-1631. doi: 10.1111/j.1467-8624.2004.00806.x

Kidd, C., Palmeri, H., \& Aslin, R. N. (2013). Rational snacking: Young children's decisionmaking on the marshmallow task is moderated by beliefs about environmental reliability. Cognition, 126(1), 109-114. doi: 10.1016/j.cognition.2012.08.004

Lareau, A. (2011). Unequal childhoods: Class, race, and family life (2nd ed.). University of California Press.

Mageau, G. A., Bureau, J. S., Ranger, F., Allen, M. P., \& Soenens, B. (2016). The role of parental achievement goals in predicting autonomy-supportive and controlling parenting. Journal of Child and Family Studies, 25(5), 1702-1711. doi: 10.1007/s10826-015-0341-1

Nachmias, M., Gunnar, M., Mangelsdorf, S., \& Buss, K. (1996). Behavioral inhibition and stress reactivity: The moderating role of attachment security. Child Development, 67(2), 508-522. doi: $\underline{10.1111 / j .1467-8624.1996 . t b 01748 . x}$

Obradovic, J., Sulik, M. J., \& Shaffer, A. (2021). Learning to let go : Parental over-engagement predicts poorer self-regulation in Kindergartners. Journal of Family Psychology. doi:

\subsection{7/fam0000838}


Prevoo, M. J., \& Tamis-LeMonda, C. S. (2017). Parenting and globalization in western countries: explaining differences in parent-child interactions. Current Opinion in Psychology, 15, 33-39. doi: 10.1016/j.copsyc.2017.02.003

Putnam, S. P., Helbig, A. L., Gartstein, M. A., Rothbart, M. K., \& Leerkes, E. (2014). Development and assessment of short and very short forms of the Infant Behavior Questionnaire-Revised. Journal of Personality Assessment, 96(4), 445-458. doi: $10.1080 / 00223891.2013 .841171$

Radloff, L. S. (1977). The CES-D scale: A self-report depression scale for research in the general population. Journal of Applied Psychological Measurement, 1, 385-401.

Roger Mills-Koonce, W., Willoughby, M. T., Zvara, B., Barnett, M., Gustafsson, H., \& Cox, M. J. (2015). Mothers' and fathers' sensitivity and children's cognitive development in lowincome, rural families. Journal of Applied Developmental Psychology, 38, 1-10. doi: 10.1016/j.appdev.2015.01.001

Saxbe, D., Goldenberg, D., \& Rossin-Slater, M. (2018). The transition to parenthood as a critical window for adult health. American Psychologist, 73(9), 1190-1200. doi: 10.1037/amp0000376

Solomon, J., \& George, C. (1996). Defining the caregiving system: Toward a theory of caregiving. Infant Mental Health Journal, 17(3), 183-197. doi: 10.1002/(SICI)10970355(199623)17:3<183::AID-IMHJ1>3.0.CO;2-Q

Sosinsky, L. S., Marakovitz, S., \& Carter, A. S. (2004). Parent-Child Interaction Rating Scales (PCIRS). Unpublished Manual. University of Massachusetts Boston.

Stahl, A. E., \& Feigenson, L. (2015). Observing the unexpected enhances infants' learning and exploration. Science, 348(6230), 91-94. doi: 10.1126/science.aaa3799

Suresh, K. (2011). An overview of randomization techniques: An unbiased assessment of outcome in clinical research. Journal of Human Reproductive Sciences, 4(1), 8. doi: 10.4103/0974-1208.82352 
Tamis-LeMonda, C. S., Briggs, R. D., McClowry, S. G., \& Snow, D. L. (2008). Challenges to the Study of African American Parenting: Conceptualization, Sampling, Research Approaches, Measurement, and Design. Parenting, 8(4), 319-358. doi: 10.1080/15295190802612599

Tamis-LeMonda, C. S., Way, N., Hughes, D., Yoshikawa, H., Kalman, R. K., \& Niwa, E. Y. (2008). Parents' goals for children: The dynamic coexistence of individualism and collectivism in cultures and individuals: Topic review. Social Development, 17(1), 183-209. doi: 10.1111/j.1467-9507.2007.00419.x

Treyvaud, K., Doyle, L. W., Lee, K. J., Ure, A., Inder, T. E., Hunt, R. W., \& Anderson, P. J. (2016). Parenting behavior at 2 years predicts school-age performance at 7 years in very preterm children. Journal of Child Psychology and Psychiatry and Allied Disciplines, 57(7), 814-821. doi: 10.1111/jcpp.12489

Tucker-Drob, E. M., \& Harden, K. P. (2012). Early childhood cognitive development and parental cognitive stimulation: evidence for reciprocal gene-environment transactions. Developmental Science, 15(2), 250-259. doi: 10.1111/j.1467-7687.2011.01121.x

Younesian, S., Eivers, A., Shahaeian, A., Sullivan, K., \& Gilmore, L. (2020). Maternal interactive beliefs and style as predictors of language development in preterm and full term children. Journal of Child Language, 215-243. doi: 10.1017/S0305000920000148 